\title{
Distance Powers and Distance Matrices of Integral Cayley Graphs over Abelian Groups
}

\author{
Walter Klotz \\ Institut für Mathematik \\ Technische Universität Clausthal, Germany \\ klotz@math.tu-clausthal.de \\ Torsten Sander \\ Fakultät für Informatik \\ Ostfalia Hochschule für angewandte Wissenschaften, Germany \\ t.sander@ostfalia.de
}

Submitted: May 21, 2012; Accepted: Nov 1, 2012; Published: Nov 8, 2012

Mathematics Subject Classification: 05C25, 05C50

\begin{abstract}
It is shown that distance powers of an integral Cayley graph over an abelian group $\Gamma$ are again integral Cayley graphs over $\Gamma$. Moreover, it is proved that distance matrices of integral Cayley graphs over abelian groups have integral spectrum.
\end{abstract}

\section{Introduction}

Eigenvalues of an undirected graph $G$ are the eigenvalues of an arbitrary adjacency matrix of $G$. General facts about graph spectra can e.g. be found in [7] or [8]. Harary and Schwenk [10] defined $G$ to be integral if all of its eigenvalues are integers. For a survey of integral graphs see [4]. In [2] the number of integral graphs on $n$ vertices is estimated. Known characterizations of integral graphs are restricted to certain graph classes, see e.g. [1], [13], or [15]. Here we concentrate on integral Cayley graphs over abelian groups and their distance powers.

Let $\Gamma$ be a finite, additive group, $S \subseteq \Gamma,-S=\{-s: s \in S\}=S$. The undirected Cayley graph over $\Gamma$ with shift set (or symbol) $S$, Cay $(\Gamma, S)$, has vertex set $\Gamma$. Vertices $a, b \in \Gamma$ are adjacent if and only if $a-b \in S$. For general properties of Cayley graphs we refer to Godsil and Royle [9] or Biggs [5]. Note that $0 \in S$ generates a loop at every vertex of Cay $(\Gamma, S)$. Many definitions of Cayley graphs exclude this case, but its inclusion saves us from sacrificing clarity of presentation later on.

In our paper [12] we proved for an abelian group $\Gamma$ that $\operatorname{Cay}(\Gamma, S)$ is integral if $S$ belongs to the Boolean algebra $B(\Gamma)$ generated by the subgroups of $\Gamma$. Our conjecture 
that the converse is true for all integral Cayley graphs over abelian groups has recently been proved by Alperin and Peterson [3].

Proposition 1. Let $\Gamma$ be a finite abelian group, $S \subseteq \Gamma,-S=S$. Then $G=\operatorname{Cay}(\Gamma, S)$ is integral if and only if $S \in B(\Gamma)$.

Let $G=(V, E)$ be an undirected graph with vertex set $V$ and edge set $E, D$ a finite set of nonnegative integers. The distance power $G^{D}$ of $G$ is an undirected graph with vertex set $V$. Vertices $x$ and $y$ are adjacent in $G^{D}$, if their distance $d(x, y)$ in $G$ belongs to $D$. We prove that if $G$ is an integral Cayley graph over the abelian group $\Gamma$, then every distance power $G^{D}$ is also an integral Cayley graph over $\Gamma$. Moreover, we show that in a very general sense distance matrices of integral Cayley graphs over abelian groups have integral spectrum. This extends an analogous result of Ilić [11] for integral circulant graphs, which are the integral Cayley graphs over cyclic groups. Finally, we show that the class of gcd-graphs, another subclass of integral Cayley graphs over abelian groups (see [13]), is also closed under distance power operations.

\section{The Boolean Algebra $B(\Gamma)$}

Let $\Gamma$ be an arbitrary finite, additive group. We collect facts about the Boolean algebra $B(\Gamma)$ generated by the subgroups of $\Gamma$.

\subsection{Atoms of $B(\Gamma)$}

Let us determine the minimal elements of $B(\Gamma)$. To this end, we consider elements of $\Gamma$ to be equivalent, if they generate the same cyclic subgroup. The equivalence classes of this relation partition $\Gamma$ into nonempty disjoint subsets. We shall call these sets atoms. The atom represented by $a \in \Gamma$, Atom $(a)$, consists of the generating elements of the cyclic group $\langle a\rangle$.

$$
\begin{aligned}
\operatorname{Atom}(a) & =\{b \in \Gamma:\langle a\rangle=\langle b\rangle\} \\
& =\left\{k a: k \in \mathbb{Z}, 1 \leqslant k \leqslant \operatorname{ord}_{\Gamma}(a), \operatorname{gcd}\left(k, \operatorname{ord}_{\Gamma}(a)\right)=1\right\} .
\end{aligned}
$$

Here, $\mathbb{Z}$ stands for the set of all integers. For a positive integer $k$ and $a \in \Gamma$ we denote as usual by $k a$ the $k$-fold sum of terms $a,(-k) a=-(k a), 0 a=0$. By $\operatorname{ord}_{\Gamma}(a)$ we mean the order of $a$ in $\Gamma$.

Each set $\operatorname{Atom}(a)$ can be obtained by removing from $\langle a\rangle$ all elements of its proper subgroups. We bear in mind that every set $S \in B(\Gamma)$ can be derived from the cyclic subgroups of $\Gamma$ by means of repeated union, intersection and complement (with respect to $\Gamma$ ). Thus we easily arrive at the following proposition [3].

Proposition 2. For an arbitrary finite group $\Gamma$ the following statements are true:

$$
\text { 1. } \operatorname{Atom}(a) \in B(\Gamma) \text { for every } a \in \Gamma \text {. }
$$


2. For no $a \in \Gamma$ there exists a nonempty proper subset of $\operatorname{Atom}(a)$ that belongs to $B(\Gamma)$.

3. Every nonempty set $S \in B(\Gamma)$ is the union of some sets $\operatorname{Atom}(a), a \in \Gamma$.

\subsection{Sums of Sets in $B(\Gamma)$}

In this subsection $\Gamma$ denotes a finite, additive, abelian group. We define the sum of nonempty subsets $S, T$ of $\Gamma$ :

$$
S+T=\{s+t: s \in S, t \in T\} .
$$

We are going to show that the sum of sets in $B(\Gamma)$ is again a set in $B(\Gamma)$.

Lemma 1. If $\Gamma$ is a finite abelian group and $a, b \in \Gamma$ then

$$
\operatorname{Atom}(a)+\operatorname{Atom}(b) \in B(\Gamma) .
$$

Proof. We know that $\Gamma$ can be represented (see Cohn [6]) as a direct sum of cyclic groups of prime power order. This can be grouped as

$$
\Gamma=\Gamma_{1} \oplus \Gamma_{2} \oplus \cdots \oplus \Gamma_{r}
$$

where $\Gamma_{i}$ is a direct sum of cyclic groups, the order of which is a power of a prime $p_{i}$, $\left|\Gamma_{i}\right|=p_{i}^{\alpha_{i}}, \alpha_{i} \geqslant 1$ for $i=1, \ldots, r$ and $p_{i} \neq p_{j}$ for $i \neq j$. Hence we can write each element $x \in \Gamma$ as an $r$-tuple $\left(x_{i}\right)$ with $x_{i} \in \Gamma_{i}$ for $i=1, \ldots, r$.

The order of $x_{i} \in \Gamma_{i}, \operatorname{ord}_{\Gamma_{i}}\left(x_{i}\right)$, is a divisor of $p_{i}^{\alpha_{i}}$. Therefore, integer factors in the $i$-th coordinate of $x$ may be reduced modulo $p_{i}^{\alpha_{i}}$. The order of $x \in \Gamma, \operatorname{ord}_{\Gamma}(x)$, is the least common multiple of the orders of its coordinates:

$$
\operatorname{ord}_{\Gamma}(x)=\operatorname{lcm}\left(\operatorname{ord}_{\Gamma_{1}}\left(x_{1}\right), \ldots, \operatorname{ord}_{\Gamma_{r}}\left(x_{r}\right)\right) \text {. }
$$

This implies that all prime divisors of $\operatorname{ord}_{\Gamma}(x)$ belong to $\left\{p_{1}, \ldots, p_{r}\right\}$.

Let $a=\left(a_{i}\right), b=\left(b_{i}\right)$ be elements of $\Gamma$. The statement of the lemma becomes trivial for $a=0$ or $b=0$. So we may assume $a \neq 0$ and $b \neq 0$. An arbitrary element $w \in \operatorname{Atom}(a)+\operatorname{Atom}(b)$ has the following form:

$$
\begin{aligned}
& w=\lambda a+\mu b, \\
& 1 \leqslant \lambda \leqslant \operatorname{ord}_{\Gamma}(a), \quad \operatorname{gcd}\left(\lambda, \operatorname{ord}_{\Gamma}(a)\right)=1 \\
& 1 \leqslant \mu \leqslant \operatorname{ord}_{\Gamma}(b), \quad \operatorname{gcd}\left(\mu, \operatorname{ord}_{\Gamma}(b)\right)=1 .
\end{aligned}
$$

We have to show $\operatorname{Atom}(w) \subseteq \operatorname{Atom}(a)+\operatorname{Atom}(b)$. To this end, we choose the integer $\nu$ with $1 \leqslant \nu \leqslant \operatorname{ord}_{\Gamma}(w), \operatorname{gcd}\left(\nu, \operatorname{ord}_{\Gamma}(w)\right)=1$, and show $\nu w \in \operatorname{Atom}(a)+\operatorname{Atom}(b)$.

Case 1. $\left(p_{1} p_{2} \cdots p_{r}\right) \mid \operatorname{ord}_{\Gamma}(w)$. 
$\operatorname{By} \operatorname{gcd}\left(\nu, \operatorname{ord}_{\Gamma}(w)\right)=1$ we know that $\nu$ has no prime divisor in $\left\{p_{1}, \ldots, p_{r}\right\}$. On the other hand all prime divisors of $\operatorname{ord}_{\Gamma}(a)$ and of $\operatorname{ord}_{\Gamma}(b)$ are in $\left\{p_{1}, \ldots, p_{r}\right\}$. This implies $\operatorname{gcd}\left(\nu, \operatorname{ord}_{\Gamma}(a)\right)=1$ and $\operatorname{gcd}\left(\nu, \operatorname{ord}_{\Gamma}(b)\right)=1$. Setting $\lambda^{\prime}=\nu \lambda$ and $\mu^{\prime}=\nu \mu$ we achieve

$$
\begin{aligned}
& \operatorname{gcd}\left(\lambda^{\prime}, \operatorname{ord}_{\Gamma}(a)\right)=1, \lambda^{\prime} a \in \operatorname{Atom}(a), \\
& \operatorname{gcd}\left(\mu^{\prime}, \operatorname{ord}_{\Gamma}(b)\right)=1, \mu^{\prime} b \in \operatorname{Atom}(b) .
\end{aligned}
$$

Now we have by (2):

$$
\nu w=\nu \lambda a+\nu \mu b=\lambda^{\prime} a+\mu^{\prime} b \in \operatorname{Atom}(a)+\operatorname{Atom}(b) .
$$

Case 2. $\left(p_{1} p_{2} \cdots p_{r}\right) \not \operatorname{ord}_{\Gamma}(w)$.

Trivially, for $w=0 \in \operatorname{Atom}(a)+\operatorname{Atom}(b)$ we have $\nu w \in \operatorname{Atom}(a)+\operatorname{Atom}(b)$. Therefore, we may assume $w \neq 0$. Without loss of generality let

$$
\left(p_{1} \cdots p_{k}\right) \mid \operatorname{ord}_{\Gamma}(w), \operatorname{gcd}\left(\operatorname{ord}_{\Gamma}(w), p_{k+1} \cdots p_{r}\right)=1,1 \leqslant k<r
$$

Now (1) and (3) imply

$$
\begin{gathered}
w=\lambda a+\mu b=\left(\lambda a_{1}+\mu b_{1}, \ldots, \lambda a_{k}+\mu b_{k}, 0, \ldots, 0\right), \\
\lambda a_{i}+\mu b_{i} \neq 0 \text { for } i=1, \ldots, k .
\end{gathered}
$$

$\operatorname{By} \operatorname{gcd}\left(\nu, \operatorname{ord}_{\Gamma}(w)\right)=1$ we know $\operatorname{gcd}\left(\nu, p_{1} \cdots p_{k}\right)=1$. If even more $\operatorname{gcd}\left(\nu, p_{1} \cdots p_{r}\right)=1$ then we deduce $\nu w \in \operatorname{Atom}(a)+\operatorname{Atom}(b)$ as in Case 1. So we may assume that $\nu$ has at least one prime divisor in $\left\{p_{k+1}, \ldots, p_{r}\right\}$. Without loss of generality let

$$
\operatorname{gcd}\left(\nu, p_{1} \cdots p_{l}\right)=1,\left(p_{l+1} \cdots p_{r}\right) \mid \nu, k \leqslant l<r .
$$

We define

$$
\nu^{\prime}=\nu+p_{1}^{\alpha_{1}} \cdots p_{l}^{\alpha_{l}} .
$$

If we observe that integer factors in the $i$-th coordinate of $w$ can be reduced modulo $p_{i}^{\alpha_{i}}$, then we see by (4): $\nu^{\prime} w=\nu w$. Moreover, (5) and the properties of $\nu$ imply $\operatorname{gcd}\left(\nu^{\prime}, p_{1} \cdots p_{r}\right)=1$. As in Case 1 we now conclude $\nu w=\nu^{\prime} w \in \operatorname{Atom}(a)+$ $\operatorname{Atom}(b)$.

Corollary 1. If $\Gamma$ is a finite abelian group with nonempty subsets $S, T \in B(\Gamma)$ then $S+T \in B(\Gamma)$.

Proof. According to Proposition 2 the sets $S$ and $T$ are unions of atoms of $B(\Gamma)$.

$$
S=\bigcup_{i=1}^{k} \operatorname{Atom}\left(a_{i}\right), \quad T=\bigcup_{j=1}^{l} \operatorname{Atom}\left(b_{j}\right) \text {. }
$$

Then we have

$$
S+T=\bigcup_{1 \leqslant i \leqslant k, 1 \leqslant j \leqslant l}\left(\operatorname{Atom}\left(a_{i}\right)+\operatorname{Atom}\left(b_{j}\right)\right) .
$$

According to Lemma 1 the sum $\operatorname{Atom}\left(a_{i}\right)+\operatorname{Atom}\left(b_{j}\right)$ is an element of $B(\Gamma)$. Therefore, (6) implies $S+T \in B(\Gamma)$. 


\section{Distance Powers and Distance Matrices}

We repeat the definition of the distance power $G^{D}$ of an undirected graph $G=(V, E)$ from the Introduction. Let $D$ be a set of nonnegative integers. The distance power $G^{D}$ has vertex set $V$. Vertices $x, y$ are adjacent in $G^{D}$, if their distance in $G$ is $d(x, y) \in D$. If $G$ is not connected, it makes sense to allow $\infty \in D$. Clearly, $G^{\emptyset}$ is the graph without edges on $V$. The edge set of $G^{\{0\}}$ consists of a single loop at every vertex of $G$. If $G$ has no loops then $G^{\{1\}}=G$.

Theorem 1. If $G=\operatorname{Cay}(\Gamma, S)$ is an integral Cayley graph over the finite abelian group $\Gamma$ and if $D$ is a set of nonnegative integers (possibly including $\infty$ ), then the distance power $G^{D}$ is also an integral Cayley graph over $\Gamma$.

Proof. If $D=\emptyset$ then $G^{D}=\operatorname{Cay}(\Gamma, \emptyset)$ is an integral Cayley graph over $\Gamma$. We now consider the case, where $D$ has only one element,

$$
D=\{d\}, \quad d \in\{0,1, \ldots, \infty\} .
$$

In several steps we define $S^{(d)} \in B(\Gamma)$ such that $G^{\{d\}}=\operatorname{Cay}\left(\Gamma, S^{(d)}\right)$ is an integral Cayley graph over $\Gamma$. If $d$ is a distance not attained in $G$, then the assertion is confirmed by $G^{\{d\}}=\operatorname{Cay}\left(\Gamma, S^{(d)}\right)$ with $S^{(d)}=\emptyset$. If $d=0$ then we achieve our goal by $S^{(0)}=\{0\}$. Suppose now that $d=\infty$ and $G$ is disconnected. If $U=\langle S\rangle$ is the subgroup generated by $S$ in $\Gamma$, then $G$ consists of disjoint subgraphs on the cosets of $U$, all of them isomorphic to $\operatorname{Cay}(U, S)$. Vertices $x, y$ in $G^{\{\infty\}}$ are adjacent if and only if they belong to different cosets of $U$, and this is true if and only if $x-y \notin U$. Therefore, we have

$$
G^{\{\infty\}}=\operatorname{Cay}\left(\Gamma, S^{(\infty)}\right) \text { with } S^{(\infty)}=\bar{U}=\Gamma \backslash U \in B(\Gamma) .
$$

Assume now that $d \geqslant 1$ is a finite distance attained between vertices $x, y$ in $G$. The sequence of vertices in a shortest path $P$ between $x$ and $y$ in $G=\operatorname{Cay}(\Gamma, S)$ has the form

$$
x, x+s_{1}, x+s_{1}+s_{2}, \ldots, x+s_{1}+\ldots+s_{d}=y, s_{i} \in S \text { for } 1 \leqslant i \leqslant d .
$$

This implies $y-x=s_{1}+\ldots+s_{d} \in d S$, where $d S$ denotes the $d$-fold sum of the set $S$. To guarantee that there is no shorter path from $x$ to $y$ than $P$ we remove from $d S$ all multiples $k S$ for $0 \leqslant k<d, 0 S=\{0\}$. Setting

$$
S^{(d)}=d S \backslash \bigcup_{0 \leqslant k<d} k S
$$

we achieve $G^{\{d\}}=\operatorname{Cay}\left(\Gamma, S^{(d)}\right)$. If $G=\operatorname{Cay}(\Gamma, S)$ is integral, then we have $S \in B(\Gamma)$ by Proposition $1, k S \in B(\Gamma)$ for every $k \geqslant 2$ by Corollary 1 , and trivially $0 S=\{0\} \in B(\Gamma)$. By (7) this implies $S^{(d)} \in B(\Gamma)$, so $G^{\{d\}}$ is an integral Cayley graph over $\Gamma$.

To complete our proof, let

$$
D=\left\{d_{1}, \ldots, d_{r}\right\} \subseteq\{0,1, \ldots, \infty\} \text { and } S^{(D)}=\bigcup_{i=1}^{r} S^{\left(d_{i}\right)} .
$$

Then we have $S^{(D)} \in B(\Gamma)$ and $G^{D}=\operatorname{Cay}\left(\Gamma, S^{(D)}\right)$ is an integral Cayley graph over $\Gamma$ by Proposition 1. 
Let $\Gamma$ be a finite additive group. A character $\psi$ of $\Gamma$ is a homomorphism from $\Gamma$ into the multiplicative group of complex numbers. An abelian group $\Gamma$ with $n$ elements has exactly $n$ distinct characters, which represent an orthogonal basis of $\mathbb{C}^{n}$ consisting of eigenvectors for every Cayley graph over $\Gamma$. More precisely, we have (see e. g. [12] or [14])

Proposition 3. Let $\psi_{1}, \ldots, \psi_{n}$ be the distinct characters of the additive abelian group $\Gamma=\left\{v_{1}, \ldots, v_{n}\right\}, S \subseteq \Gamma,-S=S$. Assume that $A=\left(a_{i, j}\right)$ is the adjacency matrix of $G=\operatorname{Cay}(\Gamma, S)$ with respect to the given ordering of the vertex set $V(G)=\Gamma$. Then the vectors $\left(\psi_{i}\left(v_{j}\right)\right)_{j=1, \ldots, n}, 1 \leqslant i \leqslant n$, constitute an orthogonal basis of $\mathbb{C}^{n}$ consisting of eigenvectors of $A$. To the eigenvector $\left(\psi_{i}\left(v_{j}\right)\right)_{j=1, \ldots, n}$ belongs the eigenvalue $\psi_{i}(S)=$ $\sum_{s \in S} \psi_{i}(s)$.

Now we define a generalized distance matrix $\operatorname{DM}(k, G)$ of a given undirected graph $G$ with vertex set $\left\{v_{1}, \ldots, v_{n}\right\}$ as follows. Let $d_{0}=0<d_{1}<\ldots<d_{r}$ be the sequence of possible distances between vertices in $G$, possibly $d_{r}=\infty$. If $k=\left(k_{0}, \ldots, k_{r}\right)$ is a vector with integral entries, then we define the entries of $\operatorname{DM}(k, G)=\left(d_{i, j}^{(k)}\right)$ for $i, j \in\{1, \ldots, n\}$ by

$$
d_{i, j}^{(k)}=k_{t}, \text { if } d\left(v_{i}, v_{j}\right)=d_{t} .
$$

The ordinary distance matrix $\operatorname{DM}(G)$ for a connected graph $G$ is established for $k=$ $(0,1, \ldots, r)$, where $r$ is the diameter of $G$.

Let $\Gamma=\left\{v_{1}, \ldots, v_{n}\right\}$ be an abelian group and consider some integral Cayley graph $G=\operatorname{Cay}(\Gamma, S)$. Any generalized distance matrix $\operatorname{DM}(k, G)$ is an integer weighted sum of the adjacency matrices of the graphs $G^{\{d\}}$ with $d \in\left\{d_{0}, d_{1}, \ldots, d_{r}\right\}$, assuming $v_{1}, \ldots, v_{n}$ as their common vertex order. To make it more precise, for $j=0, \ldots, r$ we denote by $A^{(j)}$ the adjacency matrix of the distance power $G^{\left\{d_{j}\right\}}, A^{(0)}=I_{n}$ is the $n \times n$ unit matrix. Then we have

$$
\operatorname{DM}(k, G)=k_{0} A^{(0)}+k_{1} A^{(1)}+\ldots+k_{r} A^{(r)} .
$$

By Theorem 1, all matrices $A^{(j)}, 0 \leqslant j \leqslant r$, are adjacency matrices of integral Cayley graphs over $\Gamma$. According to Proposition 3, all Cayley graphs over $\Gamma$ have a universal common basis of complex eigenvectors. As a result, integrality extends to $\operatorname{DM}(k, G)$. This proves the following theorem.

Theorem 2. Let $G=\operatorname{Cay}(\Gamma, S)$ be an integral Cayley graph over the abelian group $\Gamma$, $|\Gamma|=n$. Then every distance matrix $\operatorname{DM}(k, G)$ as defined above has integral spectrum. Moreover, the characters $\psi_{1}, \ldots, \psi_{n}$ of $\Gamma$ represent an orthogonal basis of $\mathbb{C}^{n}$ consisting of eigenvectors of $\mathrm{DM}(k, G)$.

As we have seen in Theorem 1, the class of integral Cayley graphs over an abelian group is closed under distance power operations. We shall conclude this section by presenting a subclass which has the same closure property.

We introduce the class of $g c d$-graphs as in [13]. To this end, let the finite abelian group $\Gamma$ be represented as the direct product of cyclic groups, $\Gamma=\mathbb{Z}_{m_{1}} \oplus \ldots \oplus \mathbb{Z}_{m_{r}}, m_{i} \geqslant 1$ for $i=1, \ldots, r$. Hence the elements $x \in \Gamma$ take the form of $r$-tuples.

$$
x=\left(x_{i}\right)=\left(x_{1}, \ldots, x_{r}\right), x_{i} \in \mathbb{Z}_{m_{i}}=\left\{0,1, \ldots, m_{i}-1\right\}, 1 \leqslant i \leqslant r .
$$


Addition is coordinatewise modulo $m_{i}$. For $x=\left(x_{1}, \ldots, x_{r}\right) \in \Gamma$ and $m=\left(m_{1}, \ldots, m_{r}\right)$ we define

$$
\operatorname{gcd}(x, m)=\left(\operatorname{gcd}\left(x_{1}, m_{1}\right), \ldots, \operatorname{gcd}\left(x_{r}, m_{r}\right)\right) .
$$

Here we agree upon $\operatorname{gcd}\left(0, m_{i}\right)=m_{i}$. For a divisor tuple $d=\left(d_{1}, \ldots, d_{r}\right)$ of $m, d \mid m$, we require $d_{i} \geqslant 1$ and $d_{i} \mid m_{i}$ for every $i=1, \ldots, r$. Every divisor tuple $d$ of $m$ defines an elementary gcd-set given by

$$
S_{\Gamma}(d)=\{x \in \Gamma: \operatorname{gcd}(x, m)=d\} .
$$

Clearly, the sets $S_{\Gamma}(d)$ with $d \mid m$ form a partition of the elements of $\Gamma$. We denote by $E_{\Gamma}(x)$ the unique elementary gcd-set that contains $x$, i.e. $E_{\Gamma}(x)=S_{\Gamma}(d)$ with $d=$ $\operatorname{gcd}(x, m)$. A $g c d$-set is a union of elementary gcd-sets. By construction, the elementary gcd-sets are the atoms of the Boolean algebra $B_{\text {gcd }}(\Gamma)$ consisting of all gcd-sets of $\Gamma$. According to Theorem 1 in [13], $B_{\mathrm{gcd}}(\Gamma)$ is a Boolean sub-algebra of $B(\Gamma)$. Hence by Proposition 1, all gcd-graphs $\operatorname{Cay}(\Gamma, S), S \in B_{g c d}(\Gamma)$, are integral.

Lemma 2. If $\Gamma=\mathbb{Z}_{m_{1}} \oplus \ldots \oplus \mathbb{Z}_{m_{r}}$ and $x=\left(x_{1}, \ldots, x_{r}\right) \in \Gamma$ then

$$
E_{\Gamma}(x)=E_{\mathbb{Z}_{m_{1}}}\left(x_{1}\right) \times \ldots \times E_{\mathbb{Z}_{m_{r}}}\left(x_{r}\right) .
$$

Proof. Let $m=\left(m_{1}, \ldots, m_{r}\right)$ and $d=\left(d_{1}, \ldots, d_{r}\right)=\operatorname{gcd}(x, m)$. Then we have $y=$ $\left(y_{1}, \ldots, y_{r}\right) \in E_{\Gamma}(x)$ if and only if $\operatorname{gcd}\left(y_{i}, m_{i}\right)=d_{i}$ for $i=1, \ldots, r$. This is equivalent to $y \in S_{\mathbb{Z}_{m_{1}}}\left(d_{1}\right) \times \ldots \times S_{\mathbb{Z}_{m_{r}}}\left(d_{r}\right)$, which is the same as $y \in E_{\mathbb{Z}_{m_{1}}}\left(x_{1}\right) \times \ldots \times E_{\mathbb{Z}_{m_{r}}}\left(x_{r}\right)$.

Lemma 3. For every finite abelian group $\Gamma$, any sum of its gcd-sets is again a gcd-set.

Proof. As in the proof of Corollary 1 it suffices to show that any sum of elementary gcdsets is a gcd-set. If $\Gamma$ is cyclic, then $B_{g c d}(\Gamma)=B(\Gamma)$ (see Theorem 3 in [13]) and the result follows from Lemma 1.

Now let $\Gamma=\mathbb{Z}_{m_{1}} \oplus \ldots \oplus \mathbb{Z}_{m_{r}}, m=\left(m_{1}, \ldots, m_{r}\right), r \geqslant 2$. Further let $x=\left(x_{1}, \ldots, x_{r}\right) \in$ $\Gamma, \operatorname{gcd}(x, m)=d=\left(d_{1}, \ldots, d_{r}\right)$ and let $y=\left(y_{1}, \ldots, y_{r}\right) \in \Gamma, \operatorname{gcd}(y, m)=\delta=\left(\delta_{1}, \ldots, \delta_{r}\right)$. By Lemma 2 we have

$$
E_{\Gamma}(x)+E_{\Gamma}(y)=\left(E_{\mathbb{Z}_{m_{1}}}\left(x_{1}\right)+E_{\mathbb{Z}_{m_{1}}}\left(y_{1}\right)\right) \times \ldots \times\left(E_{\mathbb{Z}_{m_{r}}}\left(x_{r}\right)+E_{\mathbb{Z}_{m_{r}}}\left(y_{r}\right)\right) .
$$

Since the cyclic case is already solved, it follows that $E_{\mathbb{Z}_{m_{i}}}\left(x_{i}\right)+E_{\mathbb{Z}_{m_{i}}}\left(y_{i}\right)$ is a gcd-set of $\mathbb{Z}_{m_{i}}$ for $i=1, \ldots, r$. Hence $E_{\mathbb{Z}_{m_{i}}}\left(x_{i}\right)+E_{\mathbb{Z}_{m_{i}}}\left(y_{i}\right)$ is a disjoint union of elementary gcd-sets $E_{\mathbb{Z}_{m_{i}}}\left(z_{1}^{(i)}\right), \ldots, E_{\mathbb{Z}_{m_{i}}}\left(z_{\varrho_{i}}^{(i)}\right)$, with $z_{j}^{(i)} \in \mathbb{Z}_{m_{i}}$ for $j=1, \ldots, \varrho_{i}$. It follows that

$$
E_{\Gamma}(x)+E_{\Gamma}(y)=\bigcup_{1 \leqslant j_{k} \leqslant \varrho_{k}, k=1, \ldots, r}\left(E_{\mathbb{Z}_{m_{1}}}\left(z_{j_{1}}^{(1)}\right) \times \ldots \times E_{\mathbb{Z}_{m_{r}}}\left(z_{j_{r}}^{(r)}\right)\right) .
$$

Writing $z^{\left(j_{1}, \ldots, j_{r}\right)}=\left(z_{j_{1}}^{(1)}, \ldots, z_{j_{r}}^{(r)}\right)$, we get by Lemma 2

$$
E_{\Gamma}(x)+E_{\Gamma}(y)=\bigcup_{1 \leqslant j_{k} \leqslant \varrho_{k}, k=1, \ldots, r} E_{\Gamma}\left(z^{\left(j_{1}, \ldots, j_{r}\right)}\right) \in B_{g c d}(\Gamma) .
$$


The following theorem is readily deduced from Lemma 3 applying the same reasoning as in the proof of Theorem 1 .

Theorem 3. If $G=\operatorname{Cay}(\Gamma, S)$ is a gcd-graph over $\Gamma=\mathbb{Z}_{m_{1}} \oplus \ldots \oplus \mathbb{Z}_{m_{r}}$ and if $D$ is a set of nonnegative integers (possibly including $\infty$ ), then the distance power $G^{D}$ is also a gcd-graph over $\Gamma$.

\section{References}

[1] Abdollahi, A., And Vatandoost, E. Which Cayley graphs are integral? Electron. J. Comb. 16(1) (2009), R122, 1-17.

[2] Ahmadi, O., Alon, N., Blake, L. F., And Shparlinski, I. E. Graphs with integral spectrum. Linear Alg. Appl. 430 (2009), 547-552.

[3] Alperin, R. C., And Peterson, B. L. Integral Sets and Cayley Graphs of Finite Groups. Electron. J. Comb. 19 (2012), \#P44, 1-12.

[4] Balinska, K., Cvetković, D., Rodosavluević, Z., Simić, S., And SteVAnović, D. A survey on integral graphs. Univ. Beograd, Publ. Elektrotehn. Fak. Ser. Mat 13 (2003), 42-65.

[5] Biggs, N. Algebraic graph theory. Second Edition. Cambridge Mathematical Library. Cambridge University Press, 1993.

[6] Cohn, P. M. Basic Algebra. Springer, London, 2003.

[7] Cvetković, D., Rowlinson, P., And Simić. S. Eigenspaces of graphs. Encyclopedia of Mathematics and its Applications, Cambridge University Press, 1997, new edition 2008.

[8] van Dam, E. R., And Haemers, W. Which graphs are determined by their spectrum? Linear Algebra Appl. 373 (2003), 241-272.

[9] Godsil, C., And Royle, G. Algebraic graph theory. Graduate Texts in Mathematics. Vol 207. Springer, 2001.

[10] Harary, F., AND Schwenk, A. J. Which graphs have integral spectra? Lect. Notes Math. 406, Springer Verlag (1974), 45-50.

[11] ILIĆ, A. Distance Spectra and Distance Energy of Integral Circulant Graphs. Linear Algebra Appl. 433 (2010), 1005-1014.

[12] Klotz, W., And SAnder, T. Integral Cayley graphs over abelian groups. Electron. J. Comb. 17 (2010), R81, 1-13.

[13] Klotz, W., And Sander, T. Integral Cayley graphs defined by greatest common divisors. Electron. J. Comb. 18 (2011), P94, 1-15.

[14] Lovász, L. Spectra of graphs with transitive groups. Periodica Mathematica Hungarica 6 (1975), 191-195.

[15] So, W. Integral circulant graphs. Discrete Math. 306 (2005), 153-158. 